\title{
The potential misidentification of Tsukamurella pulmonis as an atypical Mycobacterium species: a cautionary tale
}

\begin{abstract}
A request was made from the general practitioner of a 36-year-old lady in the community, who had a previous history of pulmonary tuberculosis, for a laboratory work-up on a sputum specimen for Mycobacterium tuberculosis. On microscopy, the specimen was Ziehl-Neelsen negative, but conventional mycobacterial culture slowly grew an organism on Löwenstein-Jensen medium, which was phenotypically determined to be an atypical Mycobacterium sp., employing routine mycobacterial conventional tests as part of the mycobacterial laboratory's standard operating protocols for the identification of Mycobacterium spp.

identification of Tsukamurella pulmonis (99\% identity; 1471/1473 bases called), with the resulting sequence being deposited in GenBank with the accession number AY741505.

The similarity of Tsukamurella to other more common pathogens expected in immunocompromised patients, such as Mycobacterium, may have previously resulted in the under-reporting of this genus (Yassin et al., 1996). Furthermore, there has been a report of the colonization of a patient with Tsukamurella subsequent to a M. tuberculosis infection (Yassin et al., 1996).
\end{abstract} The isolate was further examined employing the GenoType Mycobacterium Molecular Genetic Assay (Hain Lifescience), in accordance with the manufacturer's instructions. Although both conjugate and universal controls of the kit were functioning correctly (Fig. 1), the isolate failed to hybridize to be identified to the species level and was therefore not considered to be any of the 13 species targeted by the kit, namely Mycobacterium avium, Mycobacterium celatum, Mycobacterium chelonae, Mycobacterium fortuitum, Mycobacterium gordonae, Mycobacterium intracellulare, Mycobacterium kansasii, Mycobacterium malmoense, Mycobacterium marinum, Mycobacterium peregrinum, Mycobacterium scrofulaceum, M. tuberculosis complex or Mycobacterium xenopi. The isolate was considered to be an atypical Mycobacterium sp. In order to determine the true identification of the organism, the isolate was forwarded to the molecular section for identification employing 16S rRNA PCR and automated gene sequencing, as previously described as a standard protocol by our group (Xu et al., 2004). PCR amplifications on high-quality genomic DNA preparations of the isolate generated amplicons of the expected size and subsequent sequencing of the amplicon and sequence analysis allowed for the reliable
Laboratory detection, identification and characterization of Mycobacterium spp. in the clinical microbiology laboratory has been aided over the last 10 years through the development and introduction of several new assays, particularly molecular-based techniques. We welcome the inclusion of the Genus Control in the GenoType Mycobacterium CM Molecular Genetic Assay (Hain Lifescience), which was not present in the original assay, and which will now help in distinguishing species within the genus Mycobacterium from members of other non-mycobacterial but related genera, such as Tsukamurella.

In conclusion, care must be taken to reliably identify all acid-fast bacilli from sputum specimens which are being investigated for M. tuberculosis to exclude other acid-fast organisms from phylogenetically related genera such as Tsukamurella, particularly when the patient has had a previous history

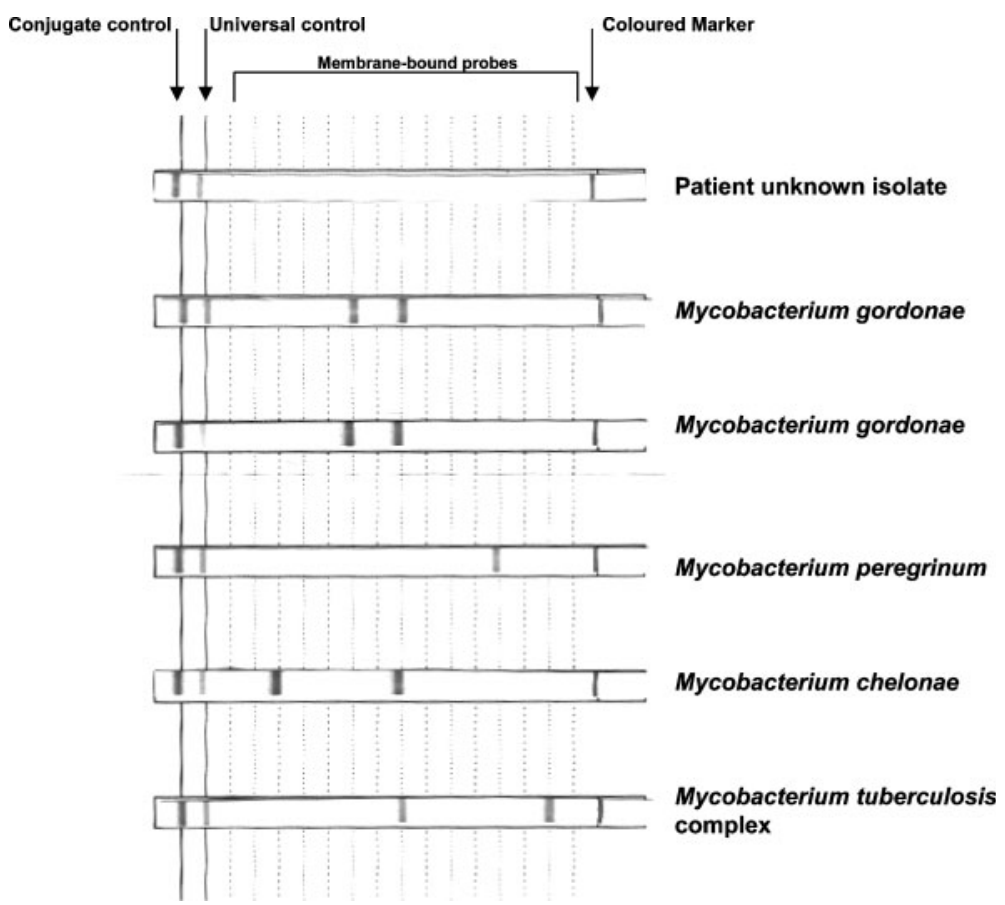

Fig. 1. Appearance of the banding profile of the unknown wild-type isolate (top lane) when employed with the GenoType Mycobacterium Molecular Genetic Assay, with control organisms M. gordonae (lanes 2 and 3), M. peregrinum (lane 4), M. chelonae (lane 5) and M. tuberculosis complex (lane 6). 
of mycobacterial disease. Help with identification of such isolates may be given by the Hain Genotype assay, in combination with 16 S rRNA PCR and automated sequencing.

Timothy Stanley, Lester Crothers, Mark McCalmont, Jiru Xu, B. Cherie Millar, Colin E. Goldsmith and John E. Moore
Northern Ireland Public Health Laboratory, Department of Bacteriology, Belfast City

Hospital, Belfast BT9 7AD, UK

Correspondence: John E. Moore

(jemoore@niphl.dnet.co.uk)

Xu, J., Smyth, C. L., Buchanan, J. A., Dolan, A., Rooney, P. J., Millar, B. C., Goldsmith, C. E.,
Elborn, J. S. \& Moore, J. E. (2004). Employment of $16 \mathrm{~S}$ rDNA gene sequencing techniques to identify culturable environmental eubacteria in a tertiary referral hospital. J Hosp Infect 57, $52-58$.

Yassin, A. F., Rainey, F. A., Brzezinka, H., Burghardt, J., Rifai, M., Seifert, P., Feldmann, K. \& Schaal, K. P. (1996). Tsukamurella pulmonis sp. nov. Int J Syst Bacteriol 46, 429-436. 